\title{
Efficiently from library to hit - crystallographic fragment screening in Berlin via structurally diverse compound libraries
}

\author{
J. Wollenhaupt ${ }^{1}$, T. Barthel' ${ }^{1}$, A. Metz ${ }^{2}$, G. M. A. Lima ${ }^{3}$, D. Wallacher ${ }^{4}$, E. Jagudin ${ }^{3}$, T. Krojer ${ }^{3}$, C. G. Feiler ${ }^{1}$, A. Heine', \\ U. Mueller ${ }^{1}$, G. Klebe ${ }^{2}$, M. S. Weiss ${ }^{1}$ \\ ${ }^{1}$ Helmholtz-Zentrum Berlin, Macromolecular Crystallography, Albert-Einstein-Str. 15, 12489 Berlin, Germany, \\ ${ }^{2}$ Philipps-Universität Marburg, Institute of Pharmaceutical Chemistry, Drug Design Group, Marbacher Weg 6, \\ 35032 Marburg, Germany, \\ ${ }^{3}$ MAX IV Laboratory, BioMAX, Fotongatan 2, 22484 Lund, Sweden, \\ ${ }^{4}$ Helmholtz-Zentrum Berlin, Department Sample Environment, Hahn-Meitner-Platz 1, 14109 Berlin, Germany
}

jan.wollenhaupt@helmholtz-berlin.de

Crystallographic fragment screening (CFS) is an established method in academia and the pharmaceutical industry thanks to dedicated workflows established and optimized at several synchrotron sites. Apart from the hit identity, this technique also provides the structural 3D-information of the fragment hits on the protein surface and therefore fosters rational tool compound development and drug discovery.

At Helmholtz-Zentrum Berlin (HZB), a dedicated CFS-workflow is available that enables stream-lined experiments and data analysis [1]. The outcome of such a workflow depends to a large extend on the quality of the fragment library employed. Higher count and chemical diversity of the resulting fragment hits increases the chances for successful design of follow-up compounds. To this end, in collaborative effort with the drug design group at Marburg University, we designed libraries that are highly diverse in terms of their 3D-pharmacophores and representative for the chemical space of fragments. The resulting F2X-Universal Library of 1103 compounds and its representative sub-selection of 96 compounds - the F2X-Entry Screen - are now the libraries of choice for CFS user campaigns at our facility due to their high performance [2]. Validation campaigns and several user campaigns were performed and showed hit rates of usually $15-25 \%$, reaching $30 \%$ in exceptional cases. Another advantage of the libraries is their physical presentation as readyto-use 96-well plates with dried-in compounds which also enables CFS for sensitive crystals without DMSO tolerance.

Apart from the exceptional library, a dedicated tool was developed at HZB to ease handling of large amounts of crystals - the EasyAccess Frame [3]. By supplying the users with such and other tools, as well as the dried-in libraries we can provide CFS campaigns as "fragment screening to go", i.e., campaigns can be conducted inside our facility or in the user's home laboratory. Furthermore, the robot assisted, and remotely controllable beamlines BL 14.1 and BL 14.2 are a key part of the HZB workflow and deliver high-quality diffraction data. Subsequently, processing of the data up to the identification of even weakly bound fragments is highly automated and provided via FragMAXapp, a user friendly, web-based tool developed in collaboration with the FragMAX facility at MAX IV [4]. Several CFS campaigns have been conducted successfully using the described workflow at HZB. As part of the EU-funded iNEXT Discovery project, application for access to the facility is straightforward and convenient for users from academia and industry.

[1] Wollenhaupt, J., Barthel, T., Lima, G.M.A., Metz, A., Wallacher, D., Jagudin, E., Huschmann, F.U., Hauß, T., Feiler, C.G., Gerlach, M., Hellmig, H., Förster, R., Steffien, M., Heine, A., Klebe, G., Mueller, U. \& Weiss, M.S. (2021). J. Vis. Exp. 169, e62208

[2] Wollenhaupt, J., Metz, A., Barthel, T., Lima, G.M.A., Heine, A., Mueller, U., Klebe, G. \& Weiss, M.S. (2020). Structure. $28,694$.

[3] Barthel, T., Huschmann, F.U., Wallacher, D., Feiler, C.G., Klebe, G., Weiss, M.S. \& Wollenhaupt, J., (2021). J. Appl. Cryst. $54,376$.

[4] Lima, G.M.A., Jagudin, E., Talibov, V.O., Benz, L.S., Costantino, M., Barthel, T., Wollenhaupt, J., Weiss, M.S. \& Mueller, U. (2021). Acta Cryst. D. accepted.

Keywords: crystallographic fragment screening; compound library; structure-based drug design 